\title{
Variabilidade da precipitação pluvial e produtividade do milho no semiárido brasileiro através da análise multivariada
}

\section{José Ricardo Ferreira LOPES ${ }^{1 *}$, Milena Pereira DANTAS ${ }^{2}$, Francisco Edson Paulo FERREIRA ${ }^{3}$}

\author{
${ }^{1}$ Programa de Pós-Graduação em Agronomia (Meteorologia Aplicada), Universidade Federal de Viçosa, Viçosa, MG, Brasil. \\ ${ }^{2}$ Programa de Pós-Graduação em Meteorologia, Instituto Nacional de Pesquisas Espaciais, São José dos Campos, SP, Brasil. \\ ${ }^{3}$ Secretaria de Agricultura e Abastecimento, Juazeiro do Norte, CE, Brasil. \\ *E-mail: agrojrfl@gmail.com
}

Recebido em junho/2018; Aceito em outubro/2018.

\begin{abstract}
RESUMO: A produtividade de diversas culturas agrícolas é intensamente dependente da oferta pluvial, bem como de sua frequência e intensidade, por isso, objetivou-se avaliar o comportamento espacial da precipitação e da produtividade do milho no semiárido brasileiro. Para isso foram utilizadas as técnicas da análise dos componentes principais (ACP) e a análise de correlação de Pearson das variáveis para o período de 1990 a 2014. Através dessas técnicas constatou-se a alta variabilidade dos dados em escala interanual, revelando as áreas mais propícias ao cultivo do milho, como também, as áreas com as menores produtividades e assim, mais afetadas pelo estresse hídrico. $\mathrm{Na}$ maior parte do território a produtividade do milho é inferior a $1000 \mathrm{~kg} \mathrm{ha}^{-1}$. A variabilidade do rendimento do milho, nas últimas décadas, está intimamente relacionada com a variabilidade climática, haja vista que se trata de uma agricultura de subsistência e em sistema sequeiro. $\mathrm{O}$ efeito das chuvas sobre a produtividade do milho foi estatisticamente positivo, ou seja, na maior parte da região semiárida brasileira, a eficiência da produção do grão é dependente das precipitações pluviais locais.
\end{abstract}

Palavras-chave: ACP, rendimento agrícola, Zea mays L.

\section{Variability of rainfall and yield of maize at the Brazilian semiarid through multivariate analysis}

\begin{abstract}
The productivity of several agricultural crops is highly dependent on the rainfall supply, as well as their frequency and intensity. Therefore, the objective was to evaluate the spatial behavior of maize precipitation and yield in the Brazilian semi-arid region. The main components analysis (PCA) and Pearson's correlation analysis of the variables for the period from 1990 to 2014 were used. Through these techniques, the high variability of the data on a year-to-year scale was verified, revealing the most important areas. Propitious to maize cultivation, as well as areas with the lowest yields and thus, more affected by water stress. In most of the territory corn yield is less than $1000 \mathrm{~kg} \mathrm{ha}^{-1}$. The variability of maize yield in the last decades is closely related to climatic variability, since it is subsistence agriculture and a dry land system. The effect of rainfall on maize productivity was statistically positive, that is, in most of the Brazilian semi-arid region, grain production efficiency is dependent on local rainfall.
\end{abstract}

Keywords: PCA, crop yield, Zea mays L.

\section{INTRODUÇÃO}

$\mathrm{O}$ estudo da variabilidade espaço-temporal de séries meteorológicas e agrícolas contribui de forma efetiva na delimitação das áreas mais propícias ao plantio/semeadura de culturas, maximizando a produtividade.

A cultura do milho (Zea mays L.), componente importante da economia no semiárido brasileiro sofre instabilidade de cultivo, causada, principalmente, pela condicionante climática e hídrica. Mesmo estando inserida neste cenário adverso, esta cultura está dispersa por todo semiárido brasileiro, dada a sua importância cultural, econômica e social, sendo explorada em diferentes condições ambientais e nos mais variados sistemas de cultivo, indo daqueles tradicionais, caracterizado pela agricultura de subsistência, até os mais modernos, que procuram explorar o máximo a potencialidade da cultura, por meio do uso de tecnologias contemporâneas de produção.

Nesse sentido, é importante identificar áreas críticas para cultivos e de menor risco à prática da agricultura, pois, mesmo sem o uso de consideráveis volumes de água, podem-se obter produções economicamente viáveis, quando se atende às exigências hídricas nos estádios de maior necessidade, principalmente nos períodos críticos da cultura (VEIGA et al., 2015).

Existem diferentes técnicas sendo usadas para diagnosticar a influência/relação da variabilidade climática sob o rendimento das principais culturas agrícolas, na tentativa de obter informações que auxiliem no processo de tomada de decisão, como os estudos de Lobell et al. (2011) e Ray et al. (2015). Outros trabalhos utilizaram técnicas de análise multivariada aplicadas a dados climáticos e de rendimento agrícola realizado por meio de análises de séries históricas (DIAS et al., 1999; CAI et al., 2013; SILVA; Da SILVA, 2016).

Diante do exposto e considerando a importância da temática, objetivou-se avaliar o comportamento espacial da precipitação e da produtividade do milho a partir da análise de 
componentes principais (ACP), seguida da análise de correlação de Pearson, no semiárido brasileiro, no período de 1990 a 2014.

\section{MATERIAL E MÉTODOS}

\section{1. Área de estudo}

A região do semiárido brasileiro, na sua delimitação de 2005, ocupava uma área de $982.563,3 \mathrm{~km}^{2}$ e incluia $89,5 \%$, do território pertence à Região Nordeste, abrangendo a maioria dos Estados nordestinos, com a exceção do Maranhão, e o Estado de Minas Gerais, situado na Região Sudeste. Sua delimitação foi baseada com base na isoieta de $800 \mathrm{~mm}$, no Índice de Aridez de Thorntwaite de 1941 (municípios com índice de até 0,50 ) e no Risco de Seca (superior a 60\%) (SILVA, 2004; CORREIA et. al, 2011; IBGE, 2016). Essa região extremamente afetada pelas ações antropogênicas, pois apresenta muitas áreas degradadas, inclusive em processo de desertificação (VIEIRA et al., 2015).

O parâmetro de maior destaque dessa região é o clima, responsável pela variação dos outros elementos que compõem as paisagens. A ele estão adaptados a vegetação e os processos de formação do relevo, com predomínio de um processo sobre outro e de acordo com a época do ano (período seco ou chuvoso).

A Figura 1 apresenta a espacialização das 330 estações pluviométricas utilizadas neste estudo na análise do comportamento espacial e temporal da precipitação e posteriormente a correlação desta variável com a produtividade agrícola do milho.

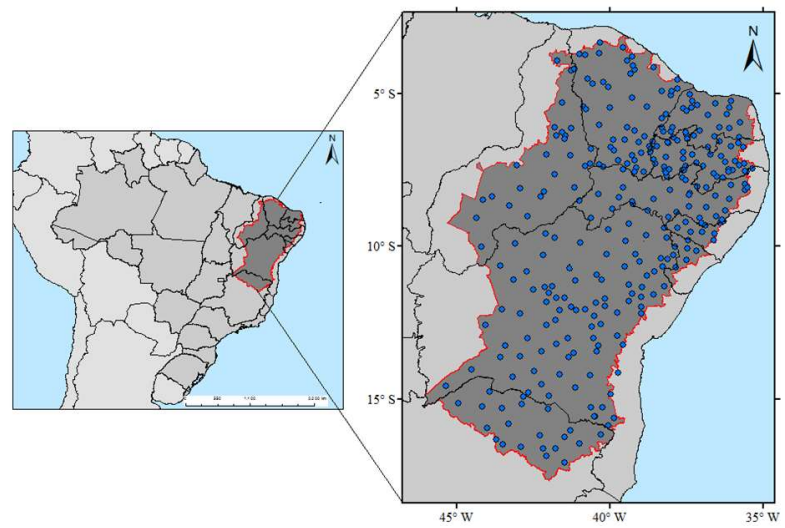

Figura 1. Mapa do Brasil com a delimitação do semiárido brasileiro e a localização dos pontos de coleta de dados.

Figure 1. Brazil Map highlighting the delimitation of the Brazilian Semi-arid region and a of the points of data base.

\subsection{Dados}

Utilizaram-se séries das médias anuais pluviométricos obtidos na Agência Nacional das Águas - ANA, na Fundação Cearense de Meteorologia e Recursos Hídricos do Estado do Ceará - FUNCEME, no Instituto Nacional de Meteorologia INMET, na Agência Executiva de Gestão das Águas do Estado da Paraíba - AESA e de produtividade média do milho (kg ha $\left.{ }^{1}\right)$ advinda do Instituto Brasileiro de Geografia e Estatística (IBGE) por meio do Sistema de Recuperação Automática SIDRA para 330 pontos distribuídos o território. Inicialmente, os dados foram analisados quanto à atualidade, ausência de falhas e consistência. Selecionou-se o período de 1990 a 2014 porque apresentou o menor número de falhas e o maior número de estações e que tivessem um período comum de observações.

\subsection{Métodos}

Aplicou-se a metodologia da análise de componentes principais (ACP) para determinar os padrões de variabilidade espacial e temporal da precipitação média anual $(\mathrm{mm})$ com a produtividade média anual do milho $\left(\mathrm{kg} \mathrm{ha}^{-1}\right)$, seguida da análise de correlação de Pearson.

A ACP é uma técnica da análise multivariada utilizada para reduzir o número de variáveis de um conjunto de dados, identificando o padrão de correlações ou de covariâncias entre elas e gerando um número menor de novas variáveis latentes a partir dos dados brutos.

A redução do número de variáveis, também chamados muitas vezes de fatores ou componentes, maximiza o poder explicativo do conjunto de todas as variáveis, a partir da classificação dos autovetores associados aos autovalores da matriz de correlação (RICHMAN, 1986; WILKS, 2006).

Pode-se dizer, então, que uma das vantagens da ACP é permitir uma simplificação estrutural do número de dados, perdendo o mínimo possível de informações dos dados originais.

Em síntese, este método consiste em transformar $p$ variáveis correlacionadas em componentes não correlacionadas ou ortogonais, tendo propriedades especiais em termos de variância. As novas componentes são combinações lineares entre autovetores e das variáveis originais normalizadas, e são mostradas em ordem decrescente de importância, ou seja, a primeira componente é a combinação linear de importância máxima (WILKS, 2006).

Seja uma matriz de $n$ observações por $m$ variáveis, onde a matriz de dados normalizada (com média zero e variância um) de dimensão $(m \mathrm{X} n)$ designada por $Z$, a partir da qual se obtém a matriz de correlação $R$ dada por:

$$
R=\frac{1}{n-1}(Z)(Z)^{\prime}
$$

(Equação 1)

em que: $Z$ ' é a matriz transposta de $Z$.

Como $R$ é a matriz simétrica positiva de dimensão $(k \mathrm{X} k)$, ela é diagonizável por uma matriz $A$, de mudança de base, denominada de autovetores e por $\lambda$, o autovalor associado, dado por:

$$
D=A^{-1} \mathrm{RA}
$$

(Equação 2)

Como os autovetores são ortogonais, a inversa de $A\left(A^{-1}\right)$ é igual a sua transposta $\left(A^{-1}\right)$. Logo as $\mathrm{CPs}_{1} \mathrm{Y}_{1}, \mathrm{Y}_{2}, \ldots, \mathrm{Y}_{\mathrm{p}}$, são obtidas por combinações lineares entre a transposta dos autovetores $\left(A^{t}\right)$ e a matriz de observação $(X)$, ou seja:

$$
\begin{aligned}
& Y=A^{t} X \\
& X=A Y
\end{aligned}
$$

Cada linha de $Y$ corresponde a uma CP, formando as séries temporais associadas aos autovalores. Os valores de $X$ do n-ésimo postos pluviométricos/estações podem ser calculados por:

$$
X_{i}=a_{j 1} Y_{1}+a_{j 2} Y_{2}+\cdots+a_{j k} Y_{k}+a_{j p} Y_{p}
$$

Cada CP contem uma porção da variância total dos dados da variável em questão (precipitação e produtividade do milho) em ordem decrescente dos autovalores mais significativos em $A$, dada pela equação: 


$$
Y_{k}=\sum_{j=1}^{m} a_{\mathrm{jk}} X_{k}
$$

(Equação 6)

$\mathrm{O}$ número de $\mathrm{CP}$ escolhido teve como base o critério de truncamento de Kaiser, que considera como mais significativos os autovalores cujos valores superam a uma unidade (GARAYALDE et al., 1986).

A distribuição espacial das CPs representa a contribuição destes em relação à variância total do conjunto de dados de precipitação e produtividade do milho em cada localidade.

Normalmente, quando a solução não rotacionadas dos fatores comuns temporais não representa de forma satisfatória a região estudada, dificultando as interpretações físicas correspondentes as CPs, então se faz a rotação dos fatores para uma nova coordenada de vetores, através de uma transformação linear. Neste estudo optou-se em fazer a rotação ortogonal VARIMAX, mantendo a correlação entre as componentes igual a zero, portanto, independentes. Logo, a variância em cada componente é dada pela equação:

$$
\operatorname{Var}=\frac{\sum_{j=1}^{r}\left(m \sum_{i-1}^{m}\left(a_{\mathrm{ij}}\right)^{2}-\left(\sum_{i=1}^{m} a_{\mathrm{ij}}^{2}\right)^{2}\right)}{m^{2}} \quad \text { (Equação 7) }
$$

em que: $r$ é o número de CPs que representam as informações físicas e $m$ o número de variáveis.

As correlações lineares entre dados de precipitação e os de produtividade agrícola para a cultura do milho para na região semiárida brasileira foram feitas através de planilha eletrônica, utilizando o método de regressão linear simples. O objetivo dessa etapa é verificar se há relação matemática comprovadamente atestada, entre a produtividade agrícola e a precipitação local, comprovando ou não se a pluviosidade influencia na agricultura da região.

O método de regressão é geralmente usado para se estudar a relação entre variáveis independentes, tentando-se obter uma relação que possa ser utilizada nas estimativas ou previsões de

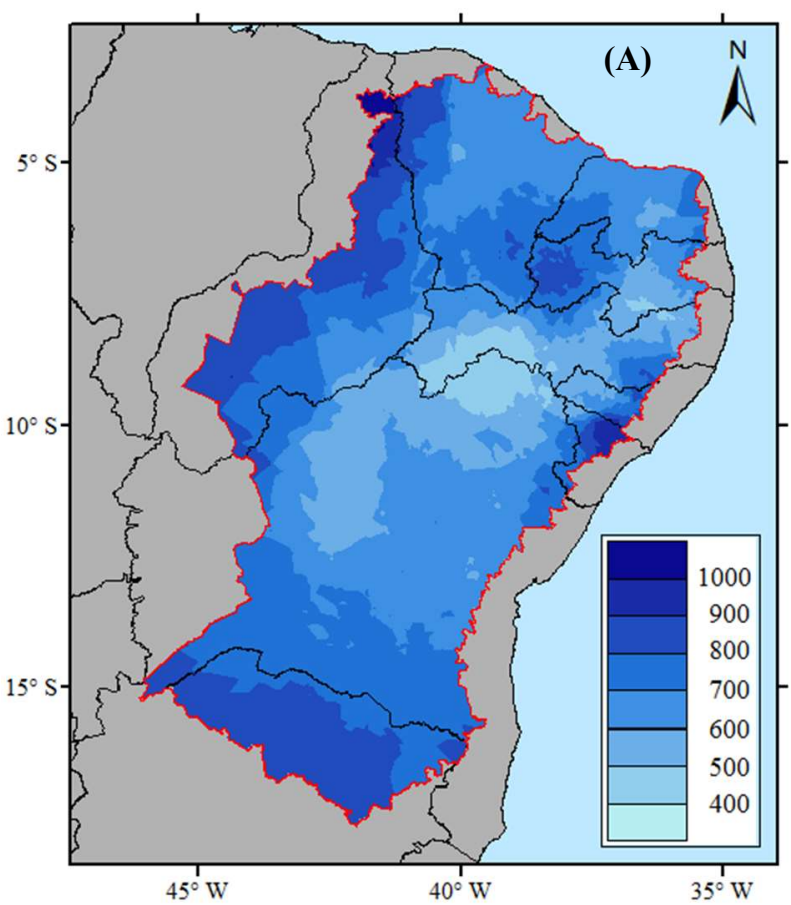

Figura 2. Distribuição espacial anual da precipitação pluvial, em mm (A) e da produtividade do milho, em $\mathrm{kg} \mathrm{ha}^{-1}$ (B), na região Semiárida brasileira, no período de 1990 a 2014. uma variável particular (CHARBA, 1975).O coeficiente de correlação de Pearson (r) é uma forma de coligação linear entre variáveis. Sua fórmula é (PEARSON, 1892):

$$
r=\frac{\sum\left(y_{i}-\bar{y}\right)(x-\bar{x})}{\sqrt{\sum\left(y_{i}-\bar{y}\right)^{2} \sum(x-\bar{x})^{2}}}
$$

em que: $x_{\mathrm{i}}$ e $y_{\mathrm{i}}$ são os valores observados de $X$ e $Y$, respectivamente; $i=1,2, \ldots, n ; n$ é o número de observações para cada variável; e $x$ e $y ́$ são as médias de $\mathrm{X}$ e $\mathrm{Y}$ respectivamente.

Para verificar a significância da correlação foi utilizado um dos testes de significância mais utilizados em estudos de meteorologia, o teste "t-Student", o qual pode ser calculado da seguinte forma:

$$
t=\frac{r \sqrt{N-2}}{\sqrt{1-r^{2}}}
$$

\section{RESULTADOS}

A Figura 2A exibe a distribuição espacial dos totais médios anuais da precipitação no semiárido brasileiro, no período de 1990 a 2014. As maiores chuvas, em torno de 900 mm, são observadas na faixa leste e oeste da região e à medida que se vai adentrando para o centro, as chuvas vão diminuindo, atingindo valores mínimos e inferiores a $600 \mathrm{~mm}$ anuais (extremo norte da Bahia e oeste de Pernambuco).

Alguns núcleos apresentam valores próximos a $1000 \mathrm{~mm}$ na porção norte e sul da área de estudo. Nestas áreas, existem microclimas específicos, que ocorrem devido à proximidade do litoral ou presença de chapadas, serras e montanhas, a exemplificar a Chapada Diamantina, na Bahia e em algumas áreas no noroeste do Ceará, oeste e sudoeste do Piauí e norte do estado de Minas Gerais.

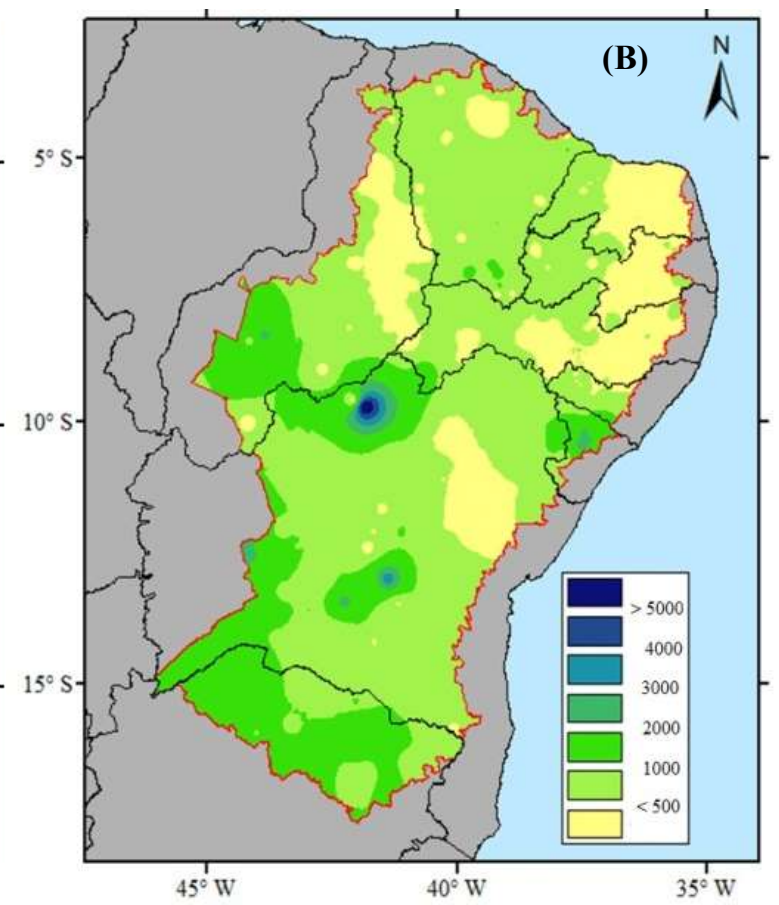

Figure 2. Annual spatial distribution of rainfall in $\mathrm{mm}(\mathrm{A})$ and maize yield, in $\mathrm{kg} \mathrm{ha}^{-1}$ (B), in the Brazilian semi-arid region, from 1990 to 2014. 
$\mathrm{Na}$ Figura 2B percebe-se que os maiores valores de produtividade média do milho estão localizados no estado da Bahia com destaque para dois núcleos (tonalidade de azul): um ao norte onde se localizam os municípios de Sento Sé (5995,88 $\left.\mathrm{kg} \mathrm{ha}^{-1}\right)$, Baianópolis $\left(3736,36 \mathrm{~kg} \mathrm{ha}^{-1}\right)$, Mucugê $(3528,12 \mathrm{~kg}$ $\left.\mathrm{ha}^{-1}\right)$. Esses municípios encontram-se no limite do cerrado baiano, na região oeste, onde a vegetação local tem sofrido uma forte pressão de desmatamento. São áreas que passam por uma expansão da fronteira agrícola, com vasta exploração não somente da cultura do milho, mas também da soja, algodão e fruticultura (MENDONÇA, 2006).

O segundo núcleo, mais ao sul, localiza-se na Chapada Diamantina, que engloba os municípios de Parnamirim (2246,92 kg ha-1), Wanderley $\left(2065,8 \mathrm{~kg} \mathrm{ha}^{-1}\right)$ e Santana $\left(2011,52 \mathrm{~kg} \mathrm{ha}^{-1}\right)$. A principal atividade econômica dessa região é a pecuária, possuindo um dos maiores rebanhos bovino da Bahia, aliada à agricultura onde se destaca o plantio do milho, além do feijão, mandioca, etc.

Deve-se também destacar o estado de Sergipe, com produtividade em torno de $2000 \mathrm{~kg} \mathrm{ha}^{-1}$ o qual apresenta tabuleiros costeiros com grande potencial para 0 desenvolvimento da cultura do milho, conforme se tem constatado em trabalhos de competição de cultivares realizado nos municípios de Nossa Senhora das Dores e Neópolis.

O sudoeste piauiense também possui relevância na produção do grão. Essa região possui extensa fronteira agrícola favorável à expansão da área cultivada e ao aumento da produtividade. Nesta área é necessário aumentar o volume dos grãos a fim de suprir a demanda estadual, a qual vem se elevando, devido ao aumento significativo da avicultura e suinocultura, como destacado por CARDOSO et al. (2003).

Com o objetivo de fazer um estudo conjunto das duas variáveis utilizadas neste estudo (precipitação e produtividade do milho) por meio da técnica multivariada de ACP obteve-se 7 fatores comuns ou 7 componentes principais (CPs) mais significativos dos totais dos dados que explicaram quase $73 \%$ da variância total dos dados. A técnica da ACP conseguiu descrever satisfatoriamente, para um nível de 95\%, as variações dos dados originais, obtendo-se um KMO (teste de Kaiser-Meyer-Olklin) de 0,947, indicando ótima adequação da técnica aos dados.

Ainda que tenham sido escolhidos 7 fatores, pelo critério de truncamento de Kaiser, foram discutidos apenas os quatro primeiros fatores rotacionados, conforme a Figura 3, que representaram $64,573 \%$ da variabilidade dos dados. Figueiredo Filho; Silva Júnior (2010) sugeriram que, apesar de não existir um critério consensual para definir quantos fatores podem ser extraídos; a solução ótima é identificar o número mínimo de fatores que maximiza a quantidade de variância total explicada (Tabela 1).

A distribuição espacial dos escores do primeiro fator, que explica $27,692 \%$ da variância total dos dados, possui escores positivos superiores a 1,0 na porção noroeste e sul (estados do Piauí e Minas Gerais) e vão diminuindo progressivamente na direção ao central da área de estudo atingindo valores negativos, próximos de $-1,5$, indicando uma alta variabilidade dos dados quando se trata da escala interanual (Figura 3A).

O segundo fator comum, que explica $17,858 \%$ da variância total dos dados, apresenta escores negativos, inferiores a $-1,0$, na parte nordeste da região abrangendo quase que por total o estado do Rio Grande do Norte e parte da Paraíba, do Pernambuco e uma pequena parte do nordeste da Bahia. Os escores positivos, maiores que 1,0 , se concentram na parte sudoeste da região. A maior parte do território do semiárida brasileira possui escores espaciais próximos de zero para este fator, reforçando a alta variabilidade da precipitação interanual na região (Figura 3B).

O padrão espacial associado ao terceiro fator é representado pela Figura 3C, que explica 15,525\% da variância total dos dados, apresenta escores positivos superiores a 0,5 distribuídos em três núcleos: o primeiro na parte sul da região semiárida brasileira, abrangendo o sul da Bahia; o segundo compreendendo quase que por total o estado de Sergipe e uma pequena parte da Bahia e o terceiro núcleo localizado no oeste da região semiárida brasileira na divisa entre os estados do Piauí e Bahia. Este fator indica, nas áreas escuras, o território com as maiores produtividades para o cultivo do milho nesses anos, conforme mostra a Figura 2B. A espacialização do quarto fator comum que explica aproximadamente $3,497 \%$ da variância total dos dados tem contribuições positivas superiores a 0,5 , em quase todo o estado da Bahia, com alguns núcleos no estado do Piauí. Os menores são observados na porção que se estende do oeste do Ceará até leste de Pernambuco com mínimos extremos no noroeste do Rio Grande do Norte, mas precisamente na fronteira com o estado do Ceará (Figura 3D).

Visando estudar a conexão entre a precipitação anual ( $\mathrm{mm})$ e a produtividade anual do milho $\left(\mathrm{kg} \mathrm{ha}^{-1}\right)$ foram calculados os coeficientes de correlações (r) entre as variáveis. A Figura 4 exibe a distribuição espacial das correlações entre a precipitação e a produtividade do milho na região semiárida brasileira.

Tabela 1. Sequência dos autovalores, variância explicada e acumulada (\%) anual dos fatores rotacionados de precipitação (mm) e da produtividade do milho $\left(\mathrm{kg} \mathrm{ha}^{-1}\right)$.

Table 1. Sequence of eigenvalues, annual explained and accumulated variance (\%) of rotated precipitation factors ( $\mathrm{mm})$ and corn yield $(\mathrm{kg}$ $\underline{\left.\mathrm{ha}^{-1}\right)}$.

\begin{tabular}{ccccccc}
\hline \multirow{2}{*}{ Componentes Principais } & \multicolumn{3}{c}{ Fatores não Rotacionadas } & \multicolumn{2}{c}{ Fatores rotacionadas (Varimax) } \\
\cline { 2 - 6 } & Autovalor & $\begin{array}{c}\text { Variância } \\
\text { Explicada (\%) }\end{array}$ & $\begin{array}{c}\text { Variância } \\
\text { Acumulada (\%) }\end{array}$ & Autovalor & $\begin{array}{c}\text { Variância } \\
\text { Explicada (\%) }\end{array}$ & $\begin{array}{c}\text { Variância } \\
\text { Acumulada (\%) }\end{array}$ \\
\hline 1 & 17,097 & 34,195 & 34,195 & 13,846 & 27,692 & 27,692 \\
2 & 11,209 & 22,419 & 56,613 & 8,929 & 17,858 & 45,55 \\
3 & 2,524 & 5,049 & 61,662 & 7,763 & 15,525 & 61,076 \\
4 & 2,029 & 4,059 & 65,721 & 1,749 & 3,497 & 64,573 \\
5 & 1,369 & 2,738 & 68,459 & 1,618 & 3,235 & 67,808 \\
6 & 1,2 & 2,401 & 70,86 & 1,395 & 2,79 & 70,598 \\
7 & 1,051 & 2,102 & 72,962 & 1,182 & 2,364 & 72,962 \\
$\ldots$ & $\ldots$ & $\ldots$ & $\ldots$ & & \\
50 & 0,07 & 0,139 & 100 & & & \\
\hline
\end{tabular}


O efeito das chuvas sobre a produtividade do milho foi, de modo geral, estatisticamente positivo, no entanto com correlações baixas $(0,10 \leq \mathrm{r} \leq 0,40)$. Percebe-se que a maior parte do semiárida brasileira possui correlações negativas e/ou próximas de zero $(0,2 \leq \mathrm{r} \leq 0,1)$ e as maiores correlações $(0,4$ $\leq \mathrm{r} \leq 1,0)$ estão localizadas no norte da área de estudo, com os maiores núcleos nos estados do Piauí, Ceará e Rio Grande do Norte.
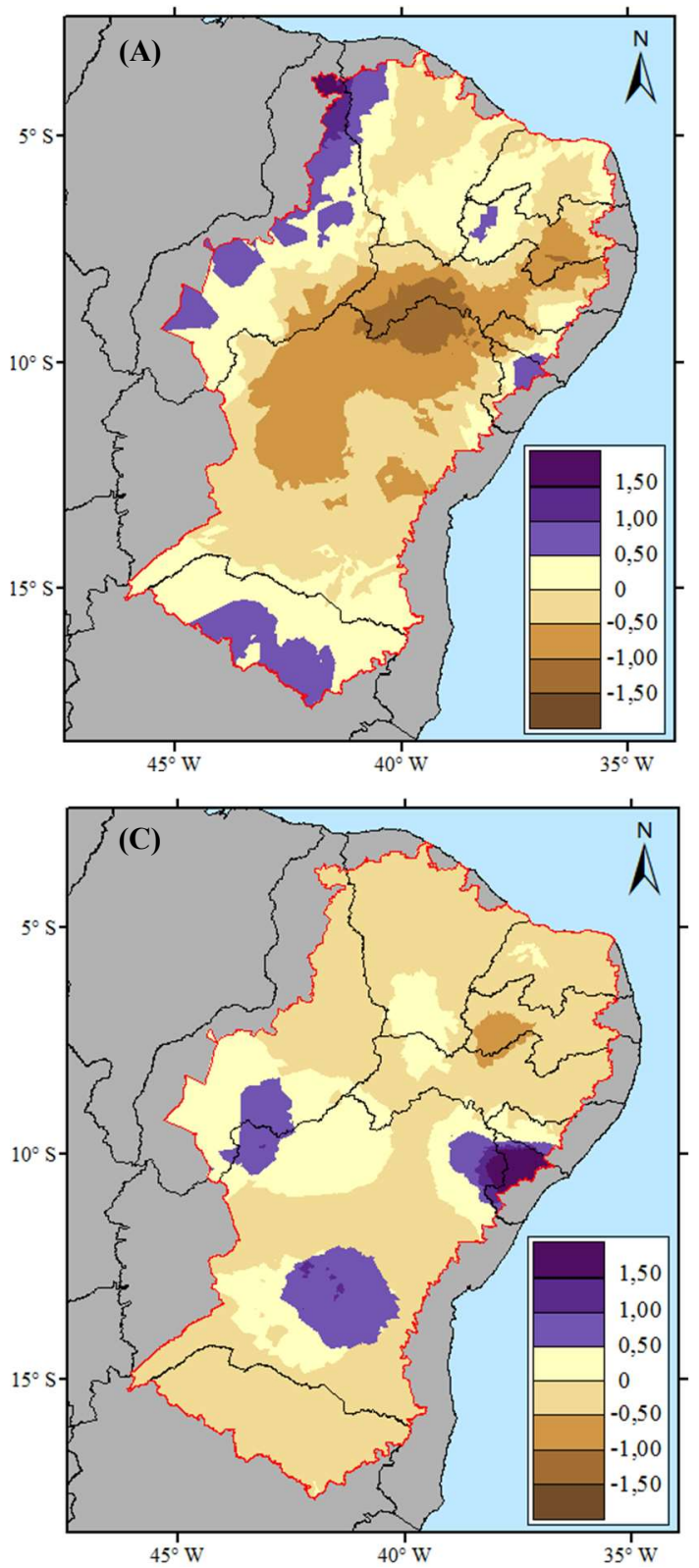

Quanto à magnitude dos coeficientes encontrados neste trabalho, deve-se levar em conta o fato de que foram correlacionados a precipitação medida na estação meteorológica e os dados estatísticos de produtividade, sendo que nestes, os fatores como épocas de semeadura, fertilidade do solo e tratos culturais não foi mantida constante.
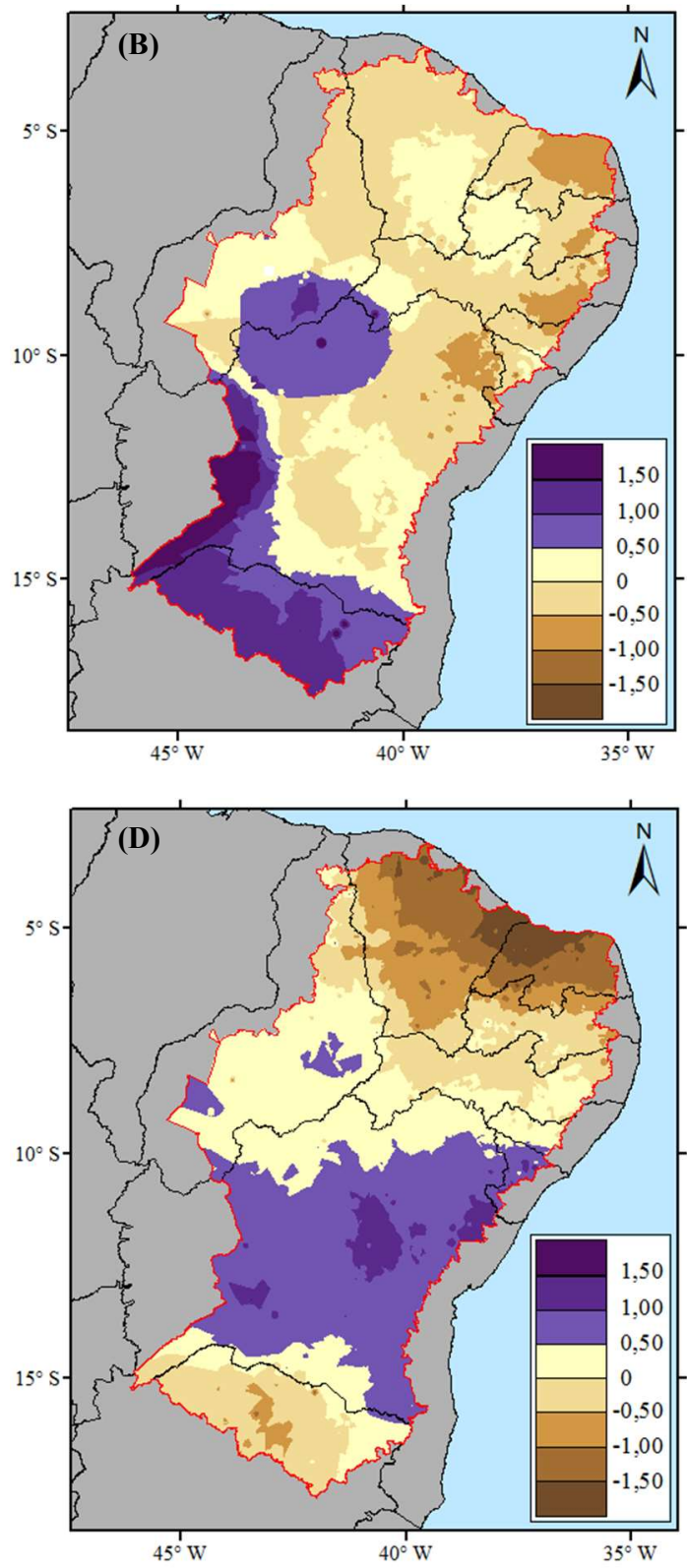

Figura 3. Distribuição espacial dos escores do primeiro (A), do segundo (B), do terceiro (C) e do quarto (D) fator comum da precipitação pluvial e da produtividade do milho na região Semiárida brasileira.

Figure 3. Spatial distribution of the first (A), second (B), third (C), and fourth (D) common rainfall precipitation and maize productivity in the Brazilian semiarid region.

\section{DISCUSSÃO}

Em quase $90 \%$ do território semiárido brasileiro a produtividade do milho é inferior a $1000 \mathrm{~kg} \mathrm{ha}^{-1}$ durante o período de 1990 a 2014 , o que configura um cultivo em sistema de sequeiro. $\mathrm{O}$ milho é um produto onipresente na cultura brasileira, no entanto, os agricultores locais não conseguem explorar a potencialidade produtiva da cultura, em virtude da alta variabilidade climática da chuva, o que condiciona baixos rendimentos do grão. Essa variabilidade espacial e interanual da chuva na região está associada a variações de padrões de Temperatura da Superfície do Mar (TSM) sobre os oceanos tropicais, o que pode interferir na atuação de sistemas atmosféricos pertencentes às várias escalas de tempo, como descrito em Ferreira et al. (2017). 
Paredes et al., 2015 sugerem que a área de influencia das anomalias relacionadas com os eventos de El Niño Oscilação Sul (ENOS) se estende do norte ao sul da região semiárida. Barbosa et al. (2016) relataram que durante a maior parte do ano, a variabilidade da precipitação local está associada a passagem da Zona de Convergência do Atlântico Sul (ZCAS), na porção sudoeste, e a atuação da Zona de Convergência Intertropical (ZCIT), na porção noroeste.

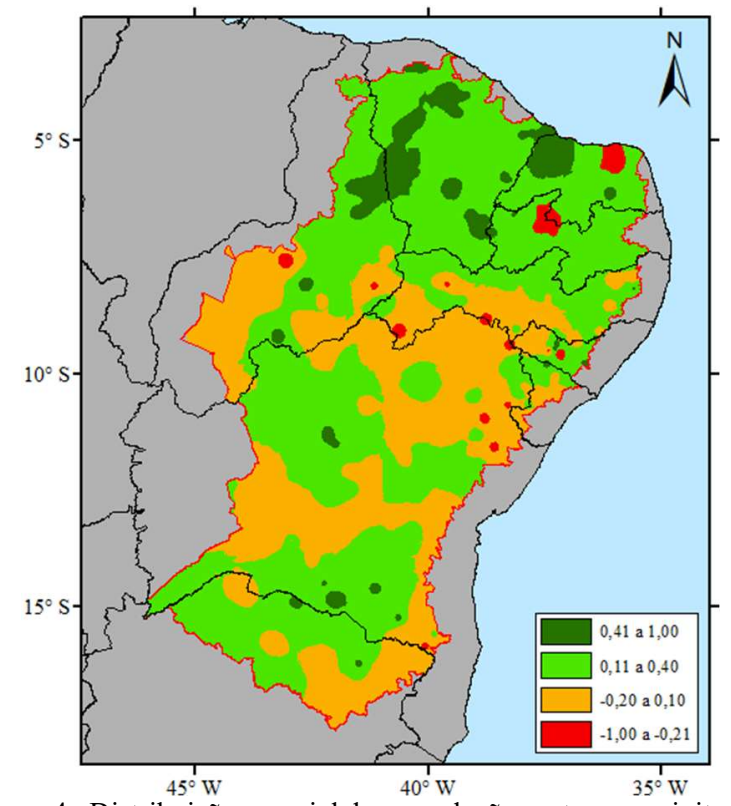

Figura 4. Distribuição espacial das correlações entre a precipitação e a produtividade do milho (1990-2014) na região semiárida brasileira. Figure 4. Spatial distribution of correlations between precipitation and maize yield (1990-2014) in the Brazilian semiarid region.

Vale salientar que o valor total anual da precipitação pode não representar muito sobre a qualidade da estação chuvosa de uma região, em especial regiões de clima semiárido, uma vez que para a agricultura, mesmo em anos em que o total de chuva é próximo ou acima da média, podem ocorrer períodos prolongados de estiagem sazonal (veranicos), que se intercalam com episódios de chuvas intensas.

Alves et al. (2017) explicam em seu estudo que esses eventos ocasionam a denominada "seca verde", que pode afetar a produção e a disponibilidade de forragem para os animais, especialmente no período sem chuvas. Essa estiagem está relacionada com a queda de produção agrícola na local, atribuindo o aumento/redução da produtividade agrícola a variabilidade do clima. Constata-se que a produtividade do milho nas últimas décadas tem sido variada e está intimamente relacionado com a variabilidade climática.

Costa (2012) analisou eventos de El Niño de acordo com sua área de atuação, classificação, ocorrências no Século XX, relacionando com as secas no Nordeste brasileiro e encontrou uma forte correlação entre o referido fenômeno e a ocorrência de seca na região Nordeste, particularmente na sua porção semiárida, oque afeta diretamente a atividade agrícola na região. Segundo Marengo et al. (2017), os eventos mais expressivos de El Niño coincidem com as secas mais prolongadas no Nordeste brasileiro. Eles demarcam ciclos que alteram as condições de tempo e clima e modificam negativamente as precipitações na Região, notadamente no polígono das secas.
Normalmente, existem dois modos básicos pelos quais a variabilidade da precipitação influencia na produtividade do milho, como apontaram Castro et al. (2016). O primeiro é que os efeitos diários, sazonais, e as variações anuais na precipitação são muito importantes para determinar a eficiência do desenvolvimento de milho. Em segundo lugar, ao considerar a exigência da chuva para o crescimento de qualquer cultura, o microclima imediatamente em torno da mesma é vital.

De modo geral, o padrão de variabilidade da chuva e da produtividade do milho é interdependente. Isso se deve as tecnologias adotadas no sistema de produção da cultura, tais como tais como melhoramento genético, variedades mais resistentes a seca, manejo de solo e o emprego da mecanização em diversos processos. Assim, não foi possível constatar estatisticamente que precipitações conduzem com os valores médios de produtividade. Contudo, pode-se afirmar que a variabilidade da precipitação interanual é um fator determinante na produtividade do grão na região.

\section{CONCLUSÕES}

A técnica estatística multivariada por meio da aplicação da análise fatorial/análise de componentes principais demonstrou, de forma eficaz e estatisticamente significante, à variabilidade da precipitação pluvial e de rendimento agrícola da cultura do milho.

A correlação entre a pluviometria e a produtividade do milho foi, de modo geral, estatisticamente positiva, no entanto, com correlações baixas. Constata-se, ainda, que a produtividade do milho nos últimos anos tem sido alterada e está intimamente relacionado com a variabilidade climática.

\section{REFERÊNCIAS}

ALVES, J. M. B.; SILVA, E. M. da; RICKES, C. P. Dynamic downscaling of precipitation and dry spells in Ceará State. Journal of Environmental Analysis and Progress, Recife, v. 2, n. 4, p. 385-393, 2017. DOI: https://doi.org/10.24221/jeap.2.4.2017.1428.385-393

BARBOSA, H. A.; LAKSHMI KUMAR, T. V. Influence of rainfall variability on the vegetation dynamics over Northeastern Brazil. Journal of Arid Environments, London, v. 124, p. 377-387, jan. 2016. DOI: http://dx.doi.org/10.1016/j.jaridenv.2015.08.015

CAI, R; MULLEN, J. D.; BERGSTROM, J. C.; W. SHURLEY, D.; WETZSTEIN, M. E. Using a Climate Index to Measure Crop Yield Response. Journal of Agricultural and Applied Economics, Auburn, v. 45, n. 4, p. 719-737, jan., 2013. DOI: https://doi.org/10.1017/S1074070800005228

CARDOSO, M. J.; CARVALHO, H. W. L.; SANTOS M. X.; LEAL. M. L. S.; OLIVEIRA, A. C. Desempenho de híbridos de milho na região Meio-Norte do Brasil. Revista Brasileira de Milho e Sorgo, Sete Lagoas, v. 2, n. 1, p. 43-52, 2003.

CASTRO, N. R.; GILIO, L.; SILVA, A. F.; OZAKI, V. A. Modelos univariados e multivariados aplicados à previsão de valores de exportação: uma análise comparativa para o complexo da soja. Espacios, Caracas, v. 37, n. 3, p. 15, 2016.

CHARBA, J. P. Operational scheme for short range forecasts of severe local weather. American Meteorological Society, Boston, p. 51-57, 1975. 
CORREIA, R. C.; KIILL, L. H. P.; MOURA, M. S. B.; CUNHA, T. J. F.; JESUS JUNIOR, L.A.; ARAÚJO, J. L. P. A região semiárida brasileira. In: Tadeu Vinhas VOLTOLINI. (Org.). Produção de caprinos e ovinos no Semiárido. Petrolina: Embrapa Semiárido, 2011.p. 21-48.

COSTA, J. A. O fenômeno El Nino e as secas no nordeste do Brasil. Revista Científica do IFAL, Maceió, v. 1, n. 4, jan./jul. 2012.

DIAS, F. L. F.; MAZZA, J. A.; MATSUOKA, S.; PERECIN, D.; MAULE, R. F. Produtividade da cana-de-açúcar em relação a clima e solos da região noroeste do Estado de São Paulo. Revista Brasileira de Ciência do Solo, Viçosa, v. 23, n. 3, p. 627-634, 1999. DOI: http://dx.doi.org/10.1590/S0100-06831999000300016

FERREIRA, P. S.; GOMES, V. P.; GALVÍNCIO, J. D.; SANTOS, A. M.; SOUZA, W. M. Avaliação da tendência espaço-temporal da precipitação pluviométrica em uma região semiárida do estado de Pernambuco. Revista Brasileira de Climatologia, v. 21, p. 113-134, jul./dez. 2017.

DOI: http://dx.doi.org/10.5380/abclima.v21i0.45895

FIGUEIREDO FILHO, D. B.; SILVA JÚNIOR, J. A. Visão além do alcance: uma introdução à análise fatorial. Opinião Pública, Campinas, v. 16, n. 1, p. 160-185, 2010.

GARAYALDE, E. J. G.; Da SILVA, M. G. A.; TAVARES, A. S. Classificação mesoclimática da região sul do Brasil pela análise de Componentes Principais. In: Congresso Interamericano de Meteorologia, 1., Congresso Brasileiro de Meteorologia, 1986, Brasília. Anais..., Brasília: Sociedade Brasileira de Meteorologia, v.1, 1986. p. 119124.

IBGE_Instituto Brasileiro de Geografia e Estatística. Áreas especiais. Disponível em: $<$ http://www.ibge.gov.br/home/geociencias/geografia/se miarido.shtm?c=4>. Acesso em: 13 mai 2018.

LOBELL, D. B.; SCHLENKER, W.; COSTA-ROBERTS, J. Climate trends and global crop production since 1980. Science, Washington, v. 333, n. 6042, p.616-620, 2011. DOI: https://doi.org/10.1126/science.1204531

MARENGO, J. A.; ALVES, L. M.; ALVALA, R. C. S .; CUNHA, A. P. ; BRITO, S.; MORAES, O. L. L. Climatic characteristics of the 2010-2016 drought in the semiarid Northeast Brazil region. An. Acad. Bras. Ciênc., Rio de Janeiro, v. 90, n. 2, p. 1-13, mai. 2017. DOI: http://dx.doi.org/10.1590/0001-3765201720170206
MENDONÇA, J. O. O potencial de crescimento da produção de grãos no Oeste da Bahia. Revista Bahia Agrícola, v.7, n.2, abr. 2006.

PAREDES, F. J.; BARBOSA, H. A., GUEVARA, E. Spatial and temporal analysis of droughts in northeastern Brazil. Agriscientia, Córdoba, v. 32, n. 1, p. 57-67, mai. 2015.

PEARSON, K. The grammar of science. London, J. M. Dent and Company, 1892. $548 \mathrm{p}$.

RAY, D. K.; GERBER, J. S.; MACDONALD, G. K.; WEST, P. C. Climate variation explains a third of global crop yield variability. Nature Communication, London, v. 6, n. 5989, p. $1-9$. 2015 . DOI: https://doi.org/10.1038/ncomms6989

RICHMAN, M. B. Rotation of principal components. Journal of Climatology, v. 6, p. 293-335, 1986.

SILVA, G.; Da SILVA, D. F. Análise da influência climática sobre a produção agrícola no semiárido cearense. Revista Brasileira de Geografia Física, Recife, v. 9, n. 2, p. 643657, 2016.

SILVA, V. P. R. On climate variability in Northeast of Brazil. Journal of Arid Environments, London, v. 58, n. 4, p. 575-596, set. $2004 . \quad$ DOI: https://doi.org/10.1016/j.jaridenv.2003.12.002

VEIGA, J. P. S.; ROMANELLI, T. L.; GIMENEZ, L. M.; BUSATO, P.; MILAN, M. Energy embodiment in Brazilian agriculture: an overview of 23 crops. Scientia Agricola, Piracicaba, v. 72, n. 6, p. 471-477, nov./dez. 2015. DOI: http://dx.doi.org/10.1590/0103-9016-20150188

VIEIRA, R. M. S. P.; TOMASELLA, J.; ALVALÁ, R. C. S.; SESTINI, M. F.; AFFONSO, A. G.; RODRIGUEZ, D. A.; BARBOSA, A. A.; CUNHA, A. P. M. A.; VALLES, G. F.; CREPANI, E.; OLIVEIRA, S. B. P.; SOUZA, M. S. B.; CALIL, P. M.; CARVALHO, M. A.; VALERIANO, D. M.; CAMPELLO, F. C. B.; SANTANA, M. O. Identifying areas susceptible to desertification in the Brazilian northeast. Solid Earth, Hoboken, v. 6, p. 347-360, 2015. DOI: https://doi.org/10.5194/se-6-347-2015

WILKS, D. S. Statistical Methods in the Atmospheric Sciences. 2. ed. London: Academic Press, 2006. 649 p. 